\title{
Generalized Stochastic Processes: The Portfolio Model
}

\author{
Moawia Alghalith \\ University of the West Indies, St. Augustine, Trinidad and Tobago \\ Email: malghalith@gmail.com
}

Received March 1, 2012; revised April 3, 2012; accepted April 15, 2012

\begin{abstract}
Using the portfolio model, we introduce a general stochastic process that is not necessarily a diffusion/jump process and the random variable is not necessarily normally distributed.
\end{abstract}

Keywords: Stochastic Process; Investment; Portfolio

\section{Introduction}

The literature on stochastic processes (especially in finance) relied mainly on Levy processes such as Wiener process, Poisson process, and the Variance-Gamma process. Examples include Madan and Seneta [1], Focardo and Fabozzi [2], among many others. Much of the literature assumes a Wiener process (Brownian motion), which implies normally distributed and independent stationary increments. The Brownian motion is extensively used in stochastic finance especially in investment models (see, for example, Alghalith [3]).

However, these assumptions of diffusion/jump process and Gaussian/Poisson distribution (or any specific probability distribution) can be relaxed. That is, we can introduce a general stochastic process that is more general than the Levy process without losing significant analytical convenience. Consequently, this paper offers three major contributions. First, it relaxes the assumption of a diffusion/jump process. Secondly, it relaxes the Gaussian/Poisson distribution or any specific probability distribution. Thirdly, it provides solutions without reliance on the existing duality or variational methods. Moreover, we introduce a general model that can be applied to any specific topic.

\section{The Model}

In general, a continuous stochastic process $\left\{X_{s}\right\}_{t \leq s \leq T}$ can be written as a function of a control variable, state variables and a random variable as the following (the first two integrals can be zero)

$$
X_{T}^{\pi}=f\left(\int_{t}^{T} \pi_{s} \mathrm{~d} s, \int_{t}^{T} \boldsymbol{\theta}_{s}\left(Y_{s}\right) \mathrm{d} s, \int_{t}^{T} \xi_{s} \mathrm{~d} s\right), t \leq T,
$$

where $\pi_{s}$ is the control variable, $\boldsymbol{\theta}_{s}$ is a vector of state variables or coefficients, $Y_{s}$ is a stochastic factor, and $\xi_{s}$ is a random variable (not necessarily a Brownian motion) and thus the assumption of normal distribution (or any specific probability distribution) is not required. Moreover, in contrast to Levy processes, $f$ is not necessarily a linear (diffusion) function. In addition, we assume $X_{s}$ is admissible and progressively measurable, where $\left\{\mathcal{F}_{s}\right\}_{t \leq s \leq T}$ is the filtration.

The objective is to maximize the expected utility of $X_{T}^{\pi}$ with respect to $\pi_{t}$

$$
\max _{\pi} E\left[u\left(X_{T}^{\pi}\right) \mid \mathcal{F}_{t}\right]
$$

where $u$ is a differentiable, bounded and concave utility function. Using the method of Alghalith [4], the solution yields

$$
E\left[u^{\prime}\left(X_{T}^{\pi}\right) f_{\pi} \mid \mathcal{F}_{t}\right]=0
$$

where the subscript denote the derivatives.

Consider this exact Taylor polynomial (and suppressing the notations) (Equation (3))

Taking expectations of both sides yields (Equation (4))

$$
\begin{gathered}
u^{\prime}(.) f_{\pi}=u^{\prime} f_{\pi}+(T-t)\left[\left(u^{\prime \prime} f_{\pi}^{2}+u^{\prime} f_{\pi \pi}\right) \pi_{t}+\sum_{i}\left(u^{\prime \prime} f_{\pi} f_{\theta_{i}}+u^{\prime} f_{\pi_{\theta_{i}}}\right) \theta_{i}+\left(u^{\prime \prime} f_{\pi} f_{\xi}+u^{\prime} f_{\pi \xi}\right) \xi\right] . \\
E_{t} u^{\prime}(.) f_{\pi}=E_{t} u^{\prime} f_{\pi}+(T-t)\left\{\left(u^{\prime \prime} f_{\pi}^{2}+u^{\prime} f_{\pi \pi}\right) \pi_{t}^{*}+\sum_{i}\left(u^{\prime \prime} f_{\pi} f_{\theta_{i}}+u^{\prime} f_{\pi_{\theta_{i}}}\right) \theta_{i}+\left(u^{\prime \prime} f_{\pi} f_{\xi}+u^{\prime} f_{\pi \xi}\right) \xi\right\}=0 .
\end{gathered}
$$


Thus,

$$
\pi_{t}^{*}=-\frac{E_{t}\left[u^{\prime} f_{\pi}+(T-t)\left\{\sum_{i}\left(u^{\prime \prime} f_{\pi} f_{\theta_{i}}+u^{\prime} f_{\pi_{\theta_{i}}}\right) \theta_{i}+\left(u^{\prime \prime} f_{\pi} f_{\xi}+u^{\prime} f_{\pi \xi}\right) \xi\right\}\right]}{(T-t) E_{t}\left(u^{\prime \prime} f_{\pi}^{2}+u^{\prime} f_{\pi \pi}\right)} .
$$

\section{Example-The Investment/Consumption Model}

It is well-known that the stock price $S_{s}$ is a function of the expected return $\mu$, the volatility $\sigma$, and a random variable $\xi$

$$
S_{T}=S\left(\int_{t}^{T} \mu\left(Y_{s}\right) \mathrm{d} s, \int_{t}^{T} \sigma\left(Y_{s}\right) \mathrm{d} s, \int_{t}^{T} \xi_{s} \mathrm{~d} s\right)
$$

where $Y_{s}$ is stochastic economic factor. However, $\xi$ is not necessarily normally distributed and $S($.$) is not$ necessarily a linear function. Consequently, the wealth function is given by

$$
X_{T}^{\pi, c}=X\left(\int_{t}^{T} \pi_{s} \mathrm{~d} s, \int_{t}^{T} c_{s} \mathrm{~d} s, x, \int_{t}^{T} r_{s}\left(Y_{s}\right) \mathrm{d} s, S_{T}\right),
$$

where $\pi_{s}$ is the portfolio process, $c_{s}$ is the consumption process, $x$ is the initial wealth, $r_{s}$ is the risk-free rate of return. Thus,

$$
X_{T}^{\pi, c}=X\left(x, \int_{t}^{T} \pi_{s} \mathrm{~d} s, \int_{t}^{T} c_{s} \mathrm{~d} s, \int_{t}^{T} \mu_{s}\left(Y_{s}\right), \int_{t}^{T} r_{s}\left(Y_{s}\right) \mathrm{d} s, \int_{t}^{T} \sigma\left(Y_{s}\right) \mathrm{d} s, \int_{t}^{T} \xi_{s} \mathrm{~d} s\right)
$$

The objective is to maximize the expected utility of wealth and consumption with respect to the portfolio and consumption

$$
\max _{\pi, c} E\left[u_{1}\left(X_{T}^{\pi, c}\right)+\int_{t}^{T} u_{2}\left(c_{s}\right) \mathrm{d} s \mid \mathcal{F}_{t}\right]
$$

The solutions are

$$
\begin{gathered}
E_{t} u_{1}^{\prime}\left(X_{T}^{\pi, c}\right) X_{\pi}=0, \\
E_{t}\left[u_{1}^{\prime}\left(X_{T}^{\pi, c}\right) X_{c}+u_{2}^{\prime}\left(c_{t}^{*}\right)\right]=0 .
\end{gathered}
$$

Using an exact Taylor expansion (and suppressing the notations), we obtain

$$
\begin{aligned}
u^{\prime}(.) X_{\pi}= & u^{\prime} X_{\pi}+(T-t)\left\{\left(u^{\prime \prime} X_{\pi}^{2}+u^{\prime} X_{\pi \pi}\right) \pi_{t}^{*}+\left(u^{\prime \prime} X_{\pi} f_{c}+u^{\prime} X_{\pi c}\right) c_{t}^{*}+\left(u^{\prime \prime} X_{\pi} f_{\mu}+u^{\prime} X_{\pi \mu}\right) \mu\right. \\
& \left.+\left(u^{\prime \prime} X_{\pi} f_{r}+u^{\prime} X_{\pi r}\right) r+\left(u^{\prime \prime} X_{\pi} f_{\sigma}+u^{\prime} X_{\pi \sigma}\right) \sigma+\left(u^{\prime \prime} X_{\pi} f_{\xi}+u^{\prime} X_{\pi \xi}\right) \xi\right\} .
\end{aligned}
$$

Thus,

$$
\begin{aligned}
E_{t} u(.) X_{\pi}= & E_{t}\left[u^{\prime} X_{\pi}+(T-t)\left\{\left(u^{\prime \prime} X_{\pi}^{2}+u^{\prime} X_{\pi \pi}\right) \pi_{t}^{*}+\left(u^{\prime \prime} X_{\pi} f_{c}+u^{\prime} X_{\pi c}\right) c_{t}^{*}+\left(u^{\prime \prime} X_{\pi} f_{\mu}+u^{\prime} X_{\pi \mu}\right) \mu\right.\right. \\
& \left.\left.+\left(u^{\prime \prime} X_{\pi} f_{r}+u^{\prime} X_{\pi r}\right) r+\left(u^{\prime \prime} X_{\pi} f_{\sigma}+u^{\prime} X_{\pi \sigma}\right) \sigma+\left(u^{\prime \prime} X_{\pi} f_{\xi}+u^{\prime} X_{\pi \xi}\right) \xi\right\}\right]
\end{aligned}
$$

Therefore we can obtain expressions for the optimal portfolio and consumption

$$
\begin{gathered}
E_{t}\left[u^{\prime} X_{\pi}+(T-t)\left\{\left(u^{\prime \prime} X_{\pi} f_{c}+u^{\prime} X_{\pi c}\right) c_{t}^{*}+\left(u^{\prime \prime} X_{\pi} f_{\mu}+u^{\prime} X_{\pi \mu}\right) \mu\right.\right. \\
\pi_{t}^{*}=-\frac{\left.\left.+\left(u^{\prime \prime} X_{\pi} f_{r}+u^{\prime} X_{\pi r}\right) r+\left(u^{\prime \prime} X_{\pi} f_{\sigma}+u^{\prime} X_{\pi \sigma}\right) \sigma+\left(u^{\prime \prime} X_{\pi} f_{\xi}+u^{\prime} X_{\pi \xi}\right) \xi\right\}\right]}{(T-t) E_{t}\left[u^{\prime \prime} X_{\pi}^{2}+u^{\prime} X_{\pi \pi}\right]} \\
c_{t}^{*}=-\frac{E_{t}\left[u^{\prime} X_{\pi}+(T-t)\left\{\left(u^{\prime \prime} X_{\pi} f_{\mu}+u^{\prime} X_{\pi \mu}\right) \mu+\left(u^{\prime \prime} X_{\pi} f_{r}+u^{\prime} X_{\pi r}\right) r\right.\right.}{\left.\left.+\left(u^{\prime \prime} X_{\pi} f_{\sigma}+u^{\prime} X_{\pi \sigma}\right) \sigma+\left(u^{\prime \prime} X_{\pi} f_{\xi}+u^{\prime} X_{\pi \xi}\right) \xi\right\}\right]} \\
(T-t) E_{t}\left[\left(u^{\prime \prime} X_{\pi} f_{c}+u^{\prime} X_{\pi c}\right)\right]
\end{gathered}
$$


We can obtain explicit solutions under specific forms of the utility function. For example, under mean-variance (quadratic) preference, we can obtain explicit solutions since $u^{\prime \prime}$ is constant and $u^{\prime}$ is linear. It is worth noting that even with Levy process general explicit solutions were not provided by the literature; thus, the assumption of a Levy process does not offer a significant analytical convenience.

\section{REFERENCES}

[1] D. Madan and E. Seneta, "The Variance-Gamma (V-G)
Model for Share Market Returns," Journal of Business, Vol. 63, No. 4, 1990, pp. 511-524. doi:10.1086/296519

[2] F. Focardi and F. Fabozzi, "The Mathematics of Financial Modeling and Investment Management," Wiley E-Series, 2004.

[3] M. Alghalith, "A New Stochastic Factor Model: General Explicit Solutions," Applied Mathematics Letters, Vol. 22, No. 12, 2009, pp. 1852-1854. doi:10.1016/j.aml.2009.07.011

[4] M. Alghalith, "An Alternative Method of Stochastic Optimization: The Portfolio Model," Applied Mathematics, Vol. 2, No. 7, 2011, pp. 912-913.

doi:10.4236/am.2011.27123 